\title{
A narrative Overview About Psychosocial Characteristics as Risk Factors of a Problematic Social Networks Use
}

\author{
Elisa Wegmann ${ }^{1} \cdot$ Matthias Brand $^{1,2}$ \\ Published online: 28 October 2019 \\ (C) The Author(s) 2019
}

\begin{abstract}
Purpose of Review Social networks use disorder describes the problematic use of social networking sites and onlinecommunication applications. Social characteristic of social networks use emphasizes the special relevance of psychosocial factors and social cognitions in this context. The current review aims at summarizing findings on individual psychosocial characteristics to analyze the relevance of social processes as potential mechanisms of a social networks use disorder.

Recent Findings The empirical overview illustrates that, aside from psychopathological factors, individual psychosocial characteristics and social cognitions as well as their interactions appear to be relevant risk factors for a problematic use of social networks. However, empirical findings are mixed.

Summary We propose two hypotheses on the development of a potential social networks use disorder that might account for mixed results: the fear-driven/compensation-seeking hypothesis including compensatory use expectancies and the reward-driven hypothesis including positive use expectancies as key elements, both of which could lead to symptoms of social networks use disorder depending on interindividual differences in predisposing and reinforcing factors.
\end{abstract}

Keywords Behavioral addictions $\cdot$ Social networking sites $\cdot$ Facebook addiction $\cdot$ Internet addiction $\cdot$ Social media use $\cdot$ Uses and gratification

\section{Introduction}

The worldwide distribution of the smartphone is a success story since different estimations illustrate that one-third of the world population has mobile Internet access resulting in a distribution of 2.7 billion smartphones all over the world [1]. Some of the most relevant smartphone applications are onlinecommunication applications such as instant messenger services, social networking sites, blogs, and microblogs, often

This article is part of the Topical Collection on Technology Addiction

Matthias Brand

matthias.brand@uni-due.de

Elisa Wegmann

elisa.wegmann@uni-due.de

1 Department of General Psychology: Cognition and Center for Behavioral Addiction Research (CeBAR), University of Duisburg-Essen, Forsthausweg 2, 47057 Duisburg, Germany

2 Erwin L. Hahn Institute for Magnetic Resonance Imaging, Essen, Germany summarized as online social networks. These applications allow users to be connected and to communicate with others, to entertain oneself, or to share information, pictures, and videos $[2,3]$. The use of these applications is often associated with positive effects on different domains such as subjective selfawareness, subjective well-being, the process of identity development as well as on communication skills, which is a result of positive interactions, perceived social support, and feelings of social connectedness [4-8].

Besides these positive effects, there is growing research about negative consequences and disadvantages due to the use of online social networks such as cyberbullying, technostress, and the excessive or addictive use of those applications [9-11]. The addictive use of online-communication applications or online social networks could be defined as "being overly concerned about social networking sites [or onlinecommunication applications], to be driven by a strong motivation to $\log$ on or to use social networking sites and to devote so much time and effort [...] that it impairs other social activities, studies/job, interpersonal relationships and/or psychological health and well-being" [9, 12, p., 4045]. In the past, researchers used different terms to describe the potential addictive use of 
online social networks or online-communication applications, e.g., social networking addiction, problematic social networks use, Facebook addiction, or Internet-communication disorder as well as smartphone addiction or problematic mobile phone use $[3,9,13]$. All these terminologies focus on the addictive use of those applications, mainly emanates from the communicative exchange, the desire to stay connected, and the experienced loss of control due to the use, regardless of the device used. Since this addiction-like behavior has not been classified as clinical disorder, the termini and the definition of gaming disorder, which has been classified as disorder due to addictive behaviors in the ICD-11, have guided us. Therefore, we prefer the term social networks use disorder or even Internetcommunication disorder, since it emphasizes the view that the user is addicted to the social, communicative activity rather than to a specific application or device [14]. Communication is an important aspect regarding the use of those applications as well as further factors such as experiencing connectedness, the need to belong, self-presentation, and entertainment or procrastination [6, 15]. Guedes et al. [16] showed that usage motives such as relationship maintenance, passing time, and entertainment are related to an addictive use, which is in line with other research [17-21]. The definition as well as the main usage motives highlight the relevance of social connectedness, social cognitions, and/or social deficits as important factors involved in the development and maintenance of a social networks use disorder. Additionally, Davis [22] proposed that negative social cognitions seem to be rather a cause than a consequence of an addictive Internet use [17], which outlines the bidirectionality of potential risk factors and the symptom severity. We argue that it is important to have a closer look on those psychosocial characteristics and specific social cognitions to address the research questions focusing on the relevance of these social processes as potential mechanisms underlying the development and maintenance of a problematic social networks use. We highlight several theoretical approaches; give an overview about current empirical studies investigating the relationship between different psychosocial characteristics and symptoms of a social networks use disorder; and discuss those results in the context of the theoretical background.

\section{Theoretical Approaches to Explain the Relevance of Psychosocial Characteristics in Social Networks Use Disorder}

The uses and gratification approach by Katz et al. [23] illustrates the experienced gratification of subjective needs by the usage of media. It is based on the assumption that users experience gratification of certain needs via media/Internet use or via specific applications, which enhances the likelihood of a repeated use in a comparable situation. The needs are distinguished among the need of entertainment, need of information, need of personal identity, and need of social interaction and integration [15]. Ryan et al. [17] propose that these motives or needs may cause the use of online social networks, which could result in a habitual, addictive use mainly motivated by the desire to escape from states of negative mood as well as to feel socially integrated (see also [10]). The dual-factor model of Facebook use by Nadkarni and Hofmann [6] also differentiates two basic social needs: the need to belong as an intrinsic drive of social connectedness and the need for self-presentation as a process of impression management [24]. This is in line with the approach by Valkenburg and Peter [25], which states two competing hypotheses: the social compensation hypothesis and the rich-get-richer hypothesis. The social compensation hypothesis assumes that individuals with high social needs and at the same time low social competences have difficulties with exchanging in daily life and making friends in face-to-face interactions. Online-communication applications are therefore a possibility to compensate these deficits, which could result in an overall preference of online social interactions (see also [26]). In contrast, the rich-getricher hypothesis outlines that individuals with low social anxiety and high social competences use Internet/online social networks as additional tools for being socially connected. On this occasion, social deficits may not be the only key mechanism, but rather social needs seem to be a central factor as well. Both hypotheses stress that online social networks could trigger the expectancies to experience compensation or satisfaction of specific needs. The theoretical argumentation of compensatory Internet use by Kardefelt-Winther [27•] also argues that the Internet is used for compensation and particularly reflecting a coping strategy, which could result in an overuse. The author argues that investigating the relevance of predisposing variables against the background of usage motives and environmental factors would result in a better understanding of potentially underlying mechanisms. Therefore, interaction effects between the different constructs should be addressed more precisely [27•]. This perspective is also central to the I-PACE (Interaction of Person-Affect-CognitionExecution) model by Brand et al. [28], which describes the process of the development and maintenance of addictive behaviors such as the proposed social networks use disorder. The model outlines that specific motives and predisposing variables such psychosocial characteristics and social deficits interact with different affective and cognitive processes (e.g., cue-reactivity, craving, internet-related cognitive biases, mood management) as well as with reductions in inhibitory control and executive functions, leading to a continued use of onlinecommunication applications in certain situations. Based on conditioned learning processes and the impact of reinforcement mechanisms, the model illustrates that these interactions result in the experience of gratification and/or compensation, at least partially depending upon the stage of the addiction process (for a more detailed explanation, see [28]). 
Wrapping up, predisposing variables such as psychosocial characteristics and further social cognitions interact with each other and could lead to the gratification of social needs and to the compensation of social deficits. After summarizing empirical studies, these are combined with the theoretical assumptions to derive a model that represents the process of development and maintenance of problematic social networks use focusing on psychosocial characteristics (see Fig. 1).

\section{Empirical Overview About Psychosocial Characteristics as Risk Factors of a Social Networks Use Disorder}

The present overview briefly summarizes empirical studies about specific personality traits and psychosocial characteristics as predictors of a potential social networks use disorder. We highlight current studies, which investigated psychopathological symptoms associated with social exchange (e.g., interpersonal sensitivity, social anxiety), personality traits (e.g., shyness), the subjective perception of social integration (e.g., feelings of loneliness, perceived social support), specific needs (e.g., need to belong), and further social cognitions (e.g., fear of social isolation and fear of missing out).

Empirical research illustrates that social anxiety is consistently associated with tendencies of a problematic social networks use [19, 24, 29-37]. Xie and Karan [29] and Andreassen et al. [30] augment that especially female users with social anxiety are at higher risk to develop a problematic social networks use. A literature review by Elhai et al. [38•] highlights the bidirectional relationship between (social) anxiety and the problematic use of mobile phones showing that anxiety enhanced the risk of a problematic behavior, which could result in higher anxiety symptoms as well. Dhir et al. [31] specify that a problematic social networks use could also result in a social media fatigue, which affects social anxiety

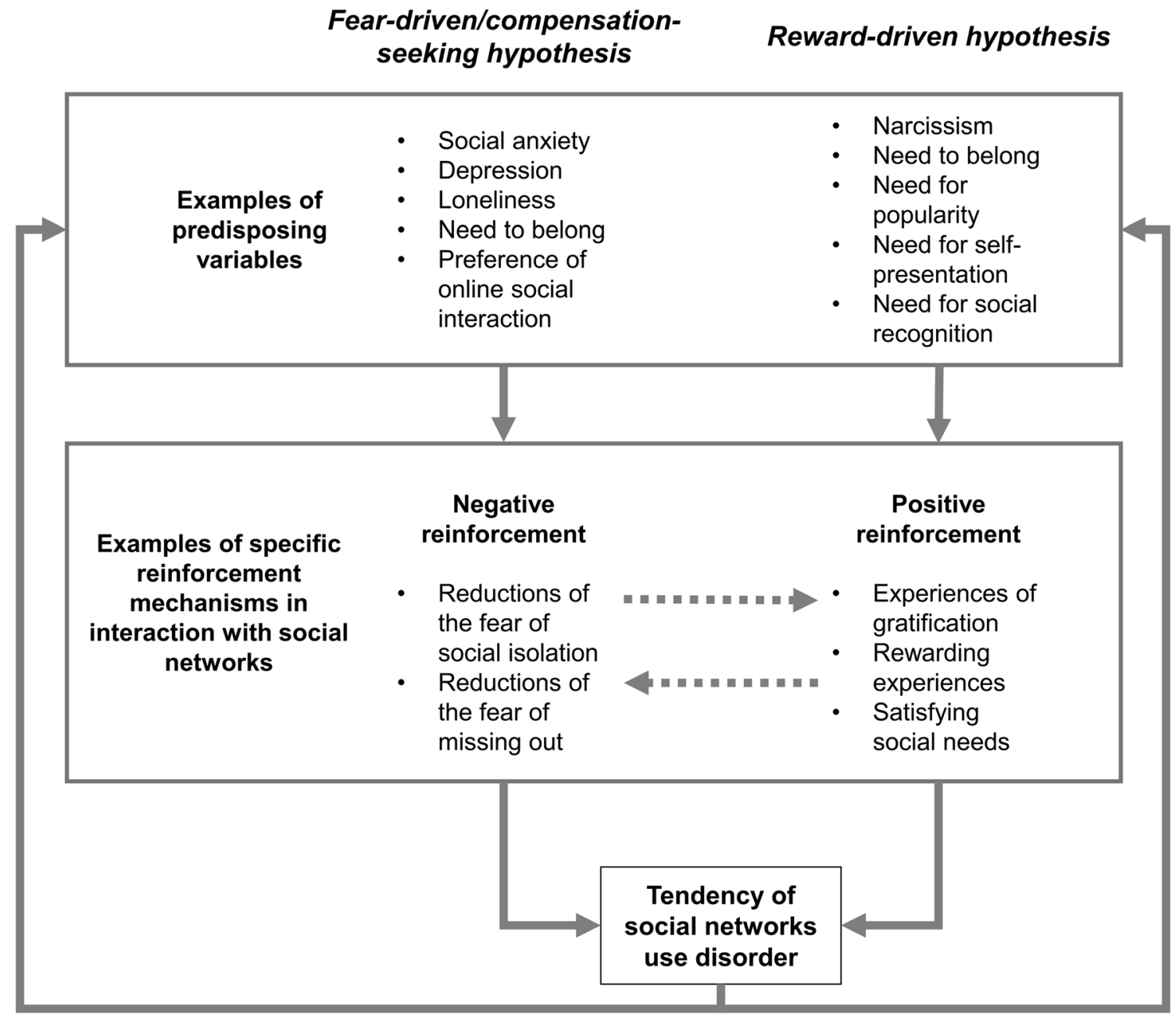

Fig. 1 This model illustrates the process of development and maintenance of a social networks use disorder focusing on psychosocial characteristics by clustering the results into two groups based on different predisposing factors and different affective and cognitive processes involved in the problematic use. The groups include either compensatory use expectancies or positive use expectancies as key elements and are referred to as fear-driven/compensation-seeking hypothesis and as reward-driven hypothesis 
and depression. Donnelly and Kuss [39] emphasized that time spent on social networks and an addictive use were associated with depressive symptoms. Interpersonal sensitivity was shown to predict symptoms of social networks use disorder, which could be explained by the desire to gratify feelings of social belongingness and social compensation [3, 40, 41]. Wegmann and Brand [3] additionally illustrated that the effect was mediated by the expectancies to avoid negative feelings or to experience pleasure.

Regarding the relevance of personality traits, Hong et al. [42] explored the relationship between different social factors and showed that the effect of shyness on symptoms of social networks use disorder was partially mediated by social anxiety. There is growing empirical evidence that shyness is related to tendencies of a potential social networks use disorder $[43,44]$. Satici [45] changed the perspective of this relationship and suggested that higher shyness and higher loneliness were consequences of tendencies of a problematic use, which also negatively affects the subjective well-being of users.

Further empirical evidence supports that individuals with higher perceived loneliness are at a higher risk of developing a so-called social networks use disorder [3, 9, 34, 46-52]. However, especially social loneliness in contrast to emotional loneliness seems to be associated with higher symptom severity, since (emotional) loneliness is not consistently related to an addictive use [3, 53-55].

Besides the role of social loneliness, Wegmann and Brand [3] showed that less perceived social support also had a direct effect on the symptom severity of a social networks use disorder. The results by Brailovskaia et al. [56] indicated that individuals perceiving high social support showed higher engagement in online social network activities and were at a higher risk to develop tendencies of a social networks use disorder. The authors differentiated between offline and online social support; therefore, high-perceived social support offline seems to be a protective factor, while online social support leads to an excessive use. Herrero et al. [57] also discussed the bivalent role of social support, since high-perceived offline social support significantly predicted higher symptom severity. However, during the addiction process, the perceived social support seems to decrease suggesting that the excessive use of online-communication application has a negative effect on the individual's social integration.

Wang et al. [58] indicated that the need to belong mediated the effect of low self-esteem and symptoms of problematic social networks use, which is consistent with that of Casale and Fioravanti [59]. The relationship between narcissism and tendencies of social networks use disorder was also mediated by the need to belong, indicating underlying psychological mechanisms of the addiction process [59]. Contradictory, the results by Casale and Fioravanti [24] illustrated that the need to belong did not mediate the effect of social anxiety on social networks use disorder. However, the need for self-presentation and the need for assertiveness were significant mediators. Furthermore, Baumeister and Leary [60] emphasized that the need to belong is a fundamental human motivation rather than a reinforcing factor.

Brand et al. [28] assume that specific reinforcement mechanisms mediate the effect of predisposing variables on the development and maintenance of addictive behaviors. The fear-of-missing-out (FoMO) construct seems to be a potential mediating factor. Several studies illustrated the importance of FoMO as a risk factor of problematic social networks use [61-69]. Furthermore, FoMO mediated the effect of specific predisposing variables (e.g., interpersonal sensitivity, depression, social anxiety, self-esteem) on tendencies of social networks use disorder $[65,66,70,71]$. In addition, further studies discussed the role of FoMO as an underlying mechanism, assuming that it is based on both social needs and the fear of social isolation [61-63]. Consequently, the fear of social isolation or of being excluded from social networks is considered a strong indicator of an addictive use of online social networks as well [72].

\section{Comparison of Theoretical Assumptions and Empirical Evidence}

The current overview illustrates the heterogeneous empirical findings regarding the relationship between psychosocial characteristics and tendencies of a potential social networks use disorder. Studies highlight both the relevance of feardriven characteristics or compensation-seeking mechanisms and the importance of positive reward expectancies combined with the experiences of gratifications or the satisfaction of specific needs. Practically speaking, the empirical studies draw a seemingly confusing picture with studies showing fear-driven mechanisms on the one hand and gratificationdriven mechanisms on the other hand, both supposed to be related to symptoms of a so-called social networks use disorder. We try to disentangle the seemingly contradictory findings by speculating that two clusters of users exist, both having a high risk to develop a problematic social networks use, but based on different predisposing factors and different affective and cognitive processes involved in the problematic use. Central to this differentiation may be the type of reinforcement mechanism interacting with specific psychosocial characteristics and usage motives as predisposing variables. The cause of the possible differentiation could be the key use expectancy, which may lead to a seemingly habitual or addictive use of social networks. We propose two hypotheses on the development of a social networks use disorder that include either compensatory use expectancies or positive use expectancies as key elements and which we refer to as fear-driven/compensation-seeking hypothesis and as reward-driven hypothesis (see Fig. 1). 


\section{The Fear-Driven/Compensation-Seeking Hypothesis}

Empirical evidence outlines that specific psychosocial characteristics, such as high social anxiety, social loneliness, and depression - referred to as social deficits in real life - are associated with higher tendencies of social networsk use disorder. Consistent with this, the high need to belong is associated with a problematic use of social networks (e.g., [16]). The preference for online social interaction in combination with low social competence but high social needs and the expectancy to reduce the feelings of fear of social isolation and FoMO may drive an excessive use of social networks. This is in line with the social compensation hypothesis, which states that unsatisfied (social) needs, social deficits, and the preference for online social interactions may result in an overuse of social networks (e.g., $[25,56])$.

Additionally, several studies showed that FoMO, avoidance expectancies when using social networks, or the anticipated fear of social isolation mediates the effect of social deficits on tendencies of a problematic social networks use (e.g., $[66,71])$. The expectancies to reduce the fear of social isolation and FoMO online could be summarized as negative reinforcement mechanism. Kardefelt-Winther [27•] also suggests that not only the association between psychopathological symptoms and tendencies of an addictive behavior are necessary to investigate but also the motivations and expectancies towards the used applications. Individuals with social deficits in real life and high social needs could have the expectancy to compensate these social deficits and to reduce the fear of social isolation by using social networks. In this fear-driven/ compensation-seeking hypothesis, we assume that the combination of low social competence, high (social) anxiety, and the negative drive of the compensation of (social) deficits describe one main motivation for engaging frequently and intensively in social networks use potentially resulting in a problematic use based on negative reinforcement mechanisms.

\section{The Reward-Driven Hypothesis}

Online-communication applications offer additional communicative possibilities, especially to meet the need of self-presentation, need for popularity, and impression management, which may play an important role in the addiction process especially for socially integrated and active individuals [6]. Mosalanejad et al. [53] showed no significant relationship between loneliness and social networks use disorder symptoms, which is inconsistent with the aforementioned fear-driven/compensation-seeking hypothesis and the results summarized above. Herrero et al. (2019) also argue that high social support enhances the risk of a problematic social networks use, which additionally negatively affects the perceived social support, which points to accumulating effects. Furthermore, Brailovskaia et al. [56] showed that individuals with high social competences may also develop a problematic behavior, which then enhances the risk of social isolation as a consequence of an excessive use. Nevertheless, at early stages, the gratification of social needs (need for popularity, need for selfpresentation) in subjects with high social competences is seemingly linked to positive reinforcement [73, 74], which is consistent with the rich-get-richer hypothesis [25], but which may revert when the behavior becomes habitual. Evidence for the rewarding character of social networks (at least in some individuals) can also be drawn from fMRI. Viewing photos on Instagram that have many likes activates the brain's reward system. In addition to receiving positive feedback in terms of likes from others, also providing feedback (likes) to others is considered rewarding and activates the brain's reward system as well [75•].

We assume that there are individuals with a high need to belong, high need for popularity, and high social competences, who have reward expectancies towards the use of online-communication applications. In this case, having an extensive social network and specific self-representative usage motives, online social networks could be used to manage and foster this network. Those applications seem to offer an additional value for the users, which could result in the expectancy to receive gratification or rewarding experiences. Individuals, who are socially integrated in real life with high egocentric motives (e.g., linked to narcissism), are at higher risk to develop a problematic behavior, when having reward expectancies towards the use of social networks and experience intense reward feelings in social interactions online. These positive reinforcement mechanisms, such as satisfying the need to belong and the experiences of gratification, characterize the reward-driven approach. However, going a step further, Guedes et al. [16] outline that the use of online social networks is associated with the expectancy to receive gratifications, which does not seem to be real, at least not for the majority of individuals. Perhaps, the absence of specific gratifications in terms of unfulfilled needs (e.g., when receiving less likes than desired) leads to a repeated usage of these applications based on the ongoing expectancy of hopefully receiving social recognition. The question remains whether the use of those online-communication applications could indeed gratify usage motives and specific needs. Therefore, longitudinal studies, which investigate the consequences of the (non-) received gratification, are needed.

\section{Conclusions and Future Directions}

We assume that individuals with a higher symptom severity of a potential social networks use disorder could be clustered into two groups based on different predisposing variables and different motivational and affective processes: (1) individuals with social deficits and fear-driven motivations to engage in 
social networks use and (2) individuals with social competence and reward-driven motivations linked to social networks use. In both cases, specific reinforcement mechanisms are crucial for the development and maintenance of a problematic social networks use. In the case of fear-driven mechanisms, we hypothesize that negative reinforcement mechanisms, such as compensatory factors, play an important role. In the case of reward-driven mechanisms, receiving gratification and the desire to receive social recognition may contribute to a seemingly addictive use. Both the fear-driven/compensation-seeking hypothesis and the reward-driven hypothesis have in common that the relationship between psychosocial characteristics, usage motives, and the tendencies of a potential social networks use disorder are likely mediated by certain processes. Those components are for example mood management, implicit associations, and cue-reactivity and craving [28], which may be further specified with respect to the fear-driven and the reward-driven hypotheses. Research illustrates that individuals with a higher tendency towards social networks use disorder experience higher craving when confronted with application-related cues [76]. However, especially craving could be an additional indicator of the two clusters, because research also differentiates between reward craving and relief craving [77]. Thus, reward craving is associated with emotionally positive experiences, which fits with the reward-driven hypothesis; relief craving is linked to reducing negative mood, which is involved in fear-driven processes.

The fear-driven/compensation-seeking hypothesis and the reward-driven hypotheses are not mutually exclusive or disjunctive. Elements of both hypotheses may interact with each other and there may also be shift from reward motivations to fear-driven processes within the addiction process, comparable to what is called the shift from gratification to compensation in other addictive behaviors [28]. This shift could also affect psychosocial characteristics and usage motives as well as the relevance of positive and negative reinforcement mechanisms. This is also in line with the conceptual framework by Elhai et al. [38•] who illustrate the bidirectional effect that anxiety can have in the context of a problematic behavior. Donnelly and Kuss [39] outline that processes of social comparison could contribute to reinforcement (intensification or stabilization) of those psychopathological symptoms as one possible consequence of the ongoing use. Therefore, possible consequences of the interaction between psychosocial characteristics, specific reinforcement mechanisms, and further key factors (e.g., cuereactivity and craving) leading to problematic social networks use should be investigated in more detail.

Acknowledgments We thank Dr. Stephanie Antons and Dr. Silke M. Müller for their valuable suggestions. Additionally, we thank Lena Kölmel and Melanie Zerr for the assistance regarding the literature search and proofreading.

\section{Compliance with Ethical Standards}

Conflict of Interest The authors declare that they have no conflict of interest.

Human and Animal Rights and Informed Consent This article does not contain any studies with human or animal subjects performed by any of the authors.

Open Access This article is distributed under the terms of the Creative Commons Attribution 4.0 International License (http:// creativecommons.org/licenses/by/4.0/), which permits unrestricted use, distribution, and reproduction in any medium, provided you give appropriate credit to the original author(s) and the source, provide a link to the Creative Commons license, and indicate if changes were made.

\section{References}

Papers of particular interest, published recently, have been highlighted as:

- Of importance

1. Statista. Number of smartphone users worldwide from 2014 to 2020 (in billions) 2017 [

2. Wu AMS, Cheung VI, Ku L, Hung EPW. Psychological risk factors of addiction to social networking sites among Chinese smartphone users. J Behav Addict. 2013;2:160-6.

3. Wegmann E, Brand M. Internet-communication disorder: it's a matter of social aspects, coping, and Internet-use expectancies. Front Psychol. 2016;7(1747):1-14.

4. Khang H, Han E-K, Ki E-J. Exploring influential social cognitive determinants of social media use. Comput Hum Behav. 2014;26: $48-55$.

5. Lee G, Lee J, Kwon S. Use of social networking sites and subjective well-being: a study in South Korea. Cyberpsychol Behav Soc Netw. 2011;14:151-5.

6. Nadkarni A, Hofmann SG. Why do people use Facebook? Personal Individ Differ. 2012;52:243-9.

7. Seabrook EM, Kern ML, Rickard NS. Social networking sites, depression, and anxiety: a systematic review. JMIR mental health. 2016;3(4):e50.

8. Allen KA, Ryan T, Gray DL, McInerney DM, Waters L. Social media use and social connectedness in adolescents: the positives and the potential pitfalls. Aust Educ Dev Psychol. 2014;31(1):1831 .

9. Andreassen CS. Online social network site addiction: a comprehensive review. Curr Addict Rep. 2015;2(2):175-84.

10. Hong F-Y, Huang DH, Lin H-Y, Chiu S-L. Analysis of the psychological traits, Facebook usage, and Facebook addiction model of Taiwanese university students. Telematics Inform. 2014;31(4):597606.

11. Livingstone S, Smith PK. Annual research review: harms experienced by child users of online and mobile technologies: the nature, prevalence and management of sexual and aggressive risks in the digital age. J Child Psychol Psychiatry. 2014.

12. Andreassen CS, Pallesen S. Social network site addiction: an overview. Curr Pharm Des. 2014;20(25):4053-61.

13. Kuss DJ, Griffiths MD. Online social networking and addiction: a review of the psychological literature. Int J Environ Res Public Health. 2011;8:3528-52. 
14. Wegmann E, Mueller SM, Ostendorf S, Brand M. Highlighting Internet-communication disorder as further Internet-use disorder when considering neuroimaging studies. Curr Behav Neurosci Rep. 2018;5(4):295-301.

15. Brandtzæg PB, Heim J. Why people use social networking sites. In: Ozok AA, Zaphiris P, editors. Online communities and social computing. Lecture Notes in Computer Science. Berlin: Springer; 2009. p. $143-152$.

16. Guedes E, Sancassiani F, Carta MG, Campos C, Machado S, King ALS, et al. Internet addiction and excessive social networks use: what about Facebook? Clin Pract Epidemiol Ment Health. 2016;12: 43-8.

17. Ryan T, Chester A, Reece J, Xenos S. The uses and abuses of Facebook: a review of Facebook addiction. J Behav Addict. 2014;3(3):133-48.

18. Giannakos MN, Chorianopoulos K, Giotopoulos K, Vlamos P. Using Facebook out of habit. Behav Inform Technol. 2013;32(6): 594-602.

19. Lee-Won RJ, Herzog L, Park SG. Hooked on Facebook: the role of social anxiety and need for social assurance in problematic use of Facebook. Cyberpsychol Behav Soc Netw. 2015;18(10):567-74.

20. Lee E, Lee JA, Moon JH, Sung Y. Pictures speak louder than words: motivations for using Instagram. Cyberpsychol Behav Soc Netw. 2015;18(9):552-6.

21. Alzougool B. The impact of motives for Facebook use on Facebook addiction among ordinary users in Jordan. Int J Soc Psyc. 2018;64(6):528-35.

22. Davis RA. A cognitive-behavioral model of pathological Internet use. Comput Hum Behav. 2001;17:187-95.

23. Katz E, Blumler GJ, Gurevich M. Utilization of mass communication by the individual. In: Blumler GJ, Katz E, editors. The uses of mass communication: Current perspectives on gratifications research. Beverly Hills, CA: Sage; 1974. p. 19-32.

24. Casale S, Fioravanti G. Satisfying needs through social networking sites: A pathway towards problematic Internet use for socially anxious people? Addict Behav Rep. 2015;1:34-9.

25. Valkenburg PM, Peter J. Preadolescents' and adolescents' online communication and their closeness to friends. Dev Psychol. 2007;43(2):267-77.

26. Caplan SE. Theory and measurement of generalized problematic Internet use: a two-step approach. Comput Hum Behav. 2010;26(5):1089-97.

27. Kardefelt-Winther D. A conceptual and methodological critique of Internet addiction research: towards a model of compensatory Internet use. Comput Hum Behav. 2014;31:351-4 This manuscript outlines a theoretical framework and defines methodological implications for investigating problematic Internet use.

28. Brand M, Wegmann E, Stark R, Müller A, Wölfling K, Robbins TW, et al. The Interaction of Person-Affect-Cognition-Execution (IPACE) model for addictive behaviors: update, generalization to addictive behaviors beyond Internet-use disorders, and specification of the process character of addictive behaviors. Neurosci Biobehav Rev. 2019.

29. Xie W, Karan K. Predicting Facebook addiction and state anxiety without Facebook by gender, trait anxiety, Facebook intensity, and different Facebook activities. J Behav Addict. 2019;8(1):79-87.

30. Andreassen CS, Billieux J, Griffiths MD, Kuss DJ, Demetrovics Z, Mazzoni E, et al. The relationship between addictive use of social media and video games and symptoms of psychiatric disorders: a large-scale cross-sectional study. Psychol Addict Behav. 2016;30(2):252-62.

31. Dhir A, Yossatorn Y, Kaur P, Chen S. Online social media fatigue and psychological wellbeing - a study of compulsive use, fear of missing out, fatigue, anxiety and depression. Int J Inf Manag. 2018;40:141-52.
32. Hussain Z, Griffiths MD, Sheffield D. An investigation into problematic smartphone use: the role of narcissism, anxiety, and personality factors. J Behav Addict. 2017;6(3):378-86.

33. Moreau A, Laconi S, Delfour M, Chabrol H. Psychopathological profiles of adolescent and young adult problematic Facebook users. Comput Hum Behav. 2015;44:64-9.

34. Yildiz Durak H, Seferoğlu SS. Modeling of variables related to problematic social media usage: social desirability tendency example. Scand J Psychol. 2019;60(3):277-88.

35. Weidman AC, Fernandez KC, Levinson CA, Augustine AA, Larsen RJ, Rodebaugh TL. Compensatory Internet use among individuals higher in social anxiety and its implications for well-being. Personal Individ Differ. 2012;53(3):191-5.

36. Lee BW, Stapinski LA. Seeking safety on the Internet: relationship between social anxiety and problematic Internet use. J Anxiety Disord. 2012;26:197-205.

37. Peterka-Bonetta J, Sindermann C, Elhai JD, Montag C. Personality associations with smartphone and Internet use disorder: a comparison study including links to impulsivity and social anxiety. Front Public Health. 2019;7:127.

38. Elhai JD, Levine JC, Hall BJ. The relationship between anxiety symptom severity and problematic smartphone use: a review of the literature and conceptual frameworks. J Anxiety Disord. 2019;62:45-52 The manuscript defines a conceptual framework based on a literature review, which illustrates the bidirectional feedback effect of predisposing variables on symptoms of a social networks use disorder.

39. Donnelly E, Kuss DJ. Depression among users of social networking sites (SNSs): the role of SNS addiction and increased usage. Addict Prev Med. 2016;1(2):1-6.

40. Eraslan-Capan B. Interpersonal sensitivity and problematic Facebook use in Turkish university students. The Anthropologist. 2015;21(3):395-403.

41. Firat S, Gul H, Sertcelik M, Gul A, Gurel Y, Kilic BG. The relationship between problematic smartphone use and psychiatric symptoms among adolescents who applied to psychiatry clinics. Psychiatry Res. 2018;270:97-103.

42. Hong W, Liu R-D, Oei T-P, Zhen R, Jiang S, Sheng X. The mediating and moderating roles of social anxiety and relatedness need satisfaction on the relationship between shyness and problematic mobile phone use among adolescents. Comput Hum Behav. 2019;93:301-8.

43. Roberts JA, Pullig C, Manolis C. I need my smartphone: a hierarchical model of personality and cell-phone addiction. Personal Individ Differ. 2015;79:13-9.

44. Ryan T, Xenos S. Who uses Facebook? An investigation into the relationship between the Big Five, shyness, narcissism, loneliness, and Facebook usage. Comput Hum Behav. 2011;27:1658-64.

45. Satici SA. Facebook addiction and subjective well-being: a study of the mediating role of shyness and loneliness. Int J Ment Health Ad. 2019;17(1):41-55.

46. Reissmann A, Hauser J, Stollberg E, Kaunzinger I, Lange KW. The role of loneliness in emerging adults' everyday use of Facebook an experience sampling approach. Comput Hum Behav. 2018;88: 47-60.

47. Suissa AJ. Cyber addictions: towards a psychosocial perspective. Addict Behav. 2015;43:28-32.

48. Omar B, Subramanian K. Addicted to Facebook: examining the roles of personality characteristics, gratification soughts and Facebook exposure among youths. Int $\mathrm{J}$ Media Commun. 2013;1(1):54-65.

49. Kim J-H. Psychological issues and problematic use of smartphone: ADHD's moderating role in the associations among loneliness, need for social assurance, need for immediate connection, and problematic use of smartphone. Comput Hum Behav. 2018;80:390-8. 
50. De Cock R, Vangeel J, Klein A, Minotte P, Rosas O, Meerkerk G-J. Compulsive use of social networking sites in Belgium: prevalence, profile, and the role of attitude toward work and school. Cyberpsychol Behav Soc Netw. 2013;17(3):166-71.

51. Kim J, LaRose R, Peng W. Loneliness as the cause and the effect of problematic Internet use: the relationship between Internet use and psychological well-being. CyberPsychology \& Behavior. 2009;12: 451-5.

52. Shettar M, Karkal R, Kakunje A, Mendonsa RD, Chandran VM. Facebook addiction and loneliness in the post-graduate students of a university in southern India. Int J Soc Psyc. 2017;63(4):325-9.

53. Mosalanejad L, Nikbakht G, Abdollahifrad S, Kalani N. The prevalence of smartphone addiction and its relationship with personality traits, loneliness and daily stress of students in Jahrom University of Medical Sciences in 2014: a cross-sectional analytical study. J Res Med Dent Sci. 2019;2:131-6.

54. Karakose T. Relationship between high school students' Facebook addiction and loneliness status. EURASIA J Math Sci Technol Educ. 2016;12(9):2419-29.

55. Biolcati R, Mancini G, Pupi V, Mugheddu V. Facebook addiction: onset predictors. J Clin Med. 2018;7(6):118.

56. Brailovskaia J, Rohmann E, Bierhoff HW, Schillack H, Margraf J. The relationship between daily stress, social support and Facebook addiction disorder. Psychiatry Res. 2019;276:167-74.

57. Herrero J, Urueña A, Torres A, Hidalgo A. Socially connected but still isolated: smartphone addiction decreases social support over time. Soc Sci Comput Rev. 2017;37(1):73-88.

58. Wang P, Zhao M, Wang X, Xie X, Wang Y, Lei L. Peer relationship and adolescent smartphone addiction: the mediating role of selfesteem and the moderating role of the need to belong. J Behav Addict. 2017;6(4):708-17.

59. Casale S, Fioravanti G. Why narcissists are at risk for developing Facebook addiction: the need to be admired and the need to belong. Addict Behav. 2018;76:312-8.

60. Baumeister RF, Leary MR. The need to belong: desire for interpersonal attachments as a fundamental human motivation. Psychol Bull. 1995;117(3):497-529.

61. Blackwell D, Leaman C, Tramposch R, Osborne C, Liss M. Extraversion, neuroticism, attachment style and fear of missing out as predictors of social media use and addiction. Personal Individ Differ. 2017;116:69-72.

62. Wolniewicz CA, Tiamiyu MF, Weeks JW, Elhai JD. Problematic smartphone use and relations with negative affect, fear of missing out, and fear of negative and positive evaluation. Psychiatry Res. 2018;262:618-23.

63. Elhai JD, Levine JC, Dvorak RD, Hall BJ. Fear of missing out, need for touch, anxiety and depression are related to problematic smartphone use. Comput Hum Behav. 2016;63:509-16.

64. Burnell K, George M, Vollet J, Ehrenreich S, Underwood M. Passive social networking site use and well-being: the mediating roles of social comparison and the fear of missing out. Cyberpsychology. 2019;13(3).
65. Oberst U, Wegmann E, Stodt B, Brand M, Chamarro A. Negative consequences from heavy social networking in adolescents: the mediating role of fear of missing out. J Adolesc. 2017;55:51-60.

66. Wegmann E, Oberst U, Stodt B, Brand M. Online-specific fear of missing out and Internet-use expectancies contribute to symptoms of Internet-communication disorder. Addict Behav Rep. 2017;5: 33-42.

67. Casale S, Rugai L, Fioravanti G. Exploring the role of positive metacognitions in explaining the association between the fear of missing out and social media addiction. Addict Behav. 2018;85: 83-7.

68. Kuss DJ, Griffiths MD. Social networking sites and addiction: ten lessons learned. Int J Environ Res Public Health. 2017;14(3):31128.

69. Al-Menayes J. The fear of missing out scale: validation of the arabic version and correlation with social media addiction. Int J Appl Psychol. 2016;2016:41-6.

70. Elhai JD, Yang H, Fang J, Bai X, Hall BJ. Depression and anxiety symptoms are related to problematic smartphone use severity in Chinese young adults: fear of missing out as a mediator. Addict Behav. 2019.

71. Dempsey AE, O'Brien KD, Tiamiyu MF, Elhai JD. Fear of missing out (FoMO) and rumination mediate relations between social anxiety and problematic Facebook use. Addict Behav Rep. 2019;9: 100150.

72. Tateno M, Teo AR, Ukai W, Kanazawa J, Katsuki R, Kubo H, et al. Internet addiction, smartphone addiction and hikikomori trait in Japanese young adult: social isolation and social network. Front Psychiatry. 2019.

73. Meshi D, Morawetz C, Heekeren H. Nucleus accumbens response to gains in reputation for the self relative to gains for others predicts social media use. Front Hum Neurosci. 2013;7(439):1-11.

74. Sherman LE, Payton AA, Hernandez LM, Greenfield PM, Dapretto $M$. The power of the like in adolescence: effects of peer influence on neural and behavioral responses to social media. Psychol Sci. 2016;27(7):1027-35.

75. Sherman LE, Hernandez LM, Greenfield PM, Dapretto M. What the brain 'likes': neural correlates of providing feedback on social media. Soc Cogn Affect Neurosci. 2018;13(7):699-707 This study investigates the neural correlates of social networking sites' features as potential rewarding mechanism.

76. Wegmann E, Stodt B, Brand M. Cue-induced craving in Internetcommunication disorder using visual and auditory cues in a cuereactivity paradigm. Addict Res Theory. 2018;26(4):306-14.

77. Verheul R, van den Brink W, Geerlings P. A three-pathway psychobiological model of craving for alcohol. Alcohol Alcohol. 1999;34(2):197-222.

Publisher's Note Springer Nature remains neutral with regard to jurisdictional claims in published maps and institutional affiliations. 